Ronilson Ferreira Freitas, 1,3 (- https://orcid org/0000-0001-9592-1774

Daniel Santos Ramos

Ohttps://orcid.org/0000-0001-8870-9660

Tahiana Ferreira Freitas

Ohttps://orid. org/0000-0002-0111-8567

Gleydson Rocha de Souza ${ }^{4}$ Ohttps://orid.org/0000-0001-6612-2658

Éryka Jovânia Pereira ${ }^{1,3}$

O https://orcid.org/0000-0003-4106-8055

Angelina do Carmo Lessa $a^{3}$ Ohttps://orcid.org/0000-0003-0708-4799

\section{Prevalência e fatores associados aos sintomas de depressão, ansiedade e estresse em professores universitários durante a pandemia da COVID-19}

\author{
Prevalence and factors associated with depression, anxiety, and stress \\ symptoms among professors during COVID-19 pandemic \\ D0I: $10.1590 / 0047-2085000000348$
}

\section{RESUMO}

Objetivo: Estimar a prevalência e os fatores associados aos sintomas da depressão, ansiedade e estresse em professores universitários da área da saúde no período da pandemia da COVID-19. Métodos: Trata-se de um estudo analítico, de caráter transversal e abordagem quantitativa com 150 professores universitários da área da saúde. Os instrumentos utilizados foram: um formulário de coleta de dados sociodemográficos, econômicos e trabalhistas. A saúde mental foi avaliada pela Escala de Depressão, Ansiedade e Estresse-21 (DASS-21). A associação entre as variáveis estudadas e a prevalência de sintomas da depressão, ansiedade e estresse foi verificada por análise bivariada seguida de regressão de Poisson, com variância robusta. Resultados: A amostra final foi composta por 150 indivíduos, sendo a média de idade de 41,4 \pm 7,9 anos, e a maioria da amostra investigada é do sexo feminino (74\%). Entre os professores, 50\% apresentaram sintomas de depressão, 37,4\% relataram sintomas de ansiedade e 47,2\% apresentaram sintomas de estresse. Após análise múltipla, observou-se que os sintomas da depressão estiveram associados à variável trabalhar em mais de uma instituição de ensino superior. As variáveis que se mostraram associadas à ansiedade foram: faixa etária $\geq 40$ anos e pessoas sem companheiro fixo. Já o estresse se mostrou associado à variável estado civil sem companheiro fixo. Conclusão: A prevalência de sintomas da depressão, ansiedade e estresse em professores universitários da área da saúde foi elevada, e fatores sociodemográficos e trabalhistas se mantiveram associados aos desfechos investigados.

\section{PALAVRAS-CHAVE}

Depressão, ansiedade, estresse, docentes, prevalência.

\begin{abstract}
Objective: To estimate the prevalence and factors associated with symptoms of depression, anxiety and stress in university professors in the health field during the pandemic period of COVID-19. Methods: This is an analytical, cross-sectional study with a quantitative approach with 150 university professors in the health field. The instruments used were: a form for collecting socio-demographic, economic and labor data. Mental health was assessed using the Depression, Anxiety and Stress Scale-21 (DASS-21). The association between the variables studied and the prevalence of symptoms of depression, anxiety and stress was verified by bivariate analysis followed by Poisson regression, with robust variance. Results: The final sample consisted of 150 individuals with a mean age of $41.4 \pm$ 7.9 years with the majority being female (74\%). 50\% of the teachers had symptoms of depression, $37.4 \%$ reported symptoms of anxiety and $47.2 \%$ had symptoms of stress. After multiple analysis, it was observed that the symptoms of depression were associated with the variable working in more than one institution of higher education. The variables that were shown to be associated with anxiety were: age group $\geq 40$ years and people without a steady partner. Stress was associated with the variable marital status without a steady partner. Conclusion: The prevalence of symptoms of depression, anxiety and stress in university professors in the health area was high, and sociodemographic and labor factors remained associated with the investigated outcomes.
\end{abstract}

KEYWORDS

Depression, anxiety, stress, teachers, prevalence. 


\section{INTRODUÇÃO}

Os problemas relacionados à saúde mental não são de diagnóstico simples, sobretudo por exigir mais do profissional médico, de sua expertise no exame clínico, do que de exames complementares. Dessa forma, é comum para os pacientes não reconhecerem seus sinais, de modo que sintomas de ansiedade e estresse podem ser negligenciados. Porém, com o passar do tempo, esses sinais e sintomas podem se agravar e, com isso, culminar em depressão, que, para cada pessoa, pode se apresentar com sintomatologias e características diferentes ${ }^{1}$. Entretanto, o que se sabe é que pessoas com depressão apresentam sintomas como desânimo, ataques de pânico, além de momentos de angústia, na qual desencadeiam o choro, e, na maioria das vezes, a própria pessoa não sabe explicar o motivo que levou a desencadeá-lo. Cada pessoa sente em uma intensidade diferente, algumas chegam a um estado crítico e acabam por tirar a própria vida ${ }^{1-3}$.

Os profissionais envolvidos em atividades em que há o contato direto com o público, como os da educação e da saúde, estão mais expostos aos riscos psicossomáticos ${ }^{4}$. Na área da educação, sintomas de depressão, ansiedade e estresse são considerados como os principais motivos de afastamento de professores, pois a cada dia esse número cresce por causa da sobrecarga e da forma como as atividades são impostas no ambiente de trabalho, fatores esses que têm contribuído para o adoecimento dos docentes ${ }^{5}$.

O termo "depressão" tem sido empregado na área da psiquiatria para designar as alterações no estado afetivo, em que o indivíduo apresenta sinais de tristeza ou ainda sintomas, síndromes e doenças ${ }^{6}$. Os principais sintomas que auxiliam no diagnóstico da depressão são humor deprimido ou irritável e interesse ou prazer diminuído em quase todas as atividades. Podem ser observados ainda, em indivíduos com síndrome depressiva, sintomas como perda ou ganho significativo de peso, insônia ou hipersonia, agitação ou retardo psicomotor, fadiga, sentimento de inutilidade ou culpa excessiva, capacidade diminuída de pensar ou concentrar-se e ideação suicida, tentativa ou plano suicida. Esse transtorno pode aparecer como uma resposta a situações reais, devido a uma reação vivencial depressiva, quando diante de fatos desagradáveis e aborrecedores, frustrações e perdas $s^{7,8}$.

A ansiedade é um estado que exprime a experiência subjetiva do indivíduo, compreendendo um conjunto de sinais e sintomas que envolvem o campo físico, emocional, mental e existencial. Trata-se de uma resposta psicológica e física à ameaça do autoconceito e caracterizada por um sentimento subjetivo de apreensão. A ansiedade pode estar associada à relação da pessoa com o ambiente ameaçador em que ela está inserida e é provocada por um aumento inesperado ou previsto de tensão ou desprazer. Destaca-se que altos níveis de ansiedade podem afetar o desempenho do indivíduo9,10.
O estresse, assim como a ansiedade, surge como consequência direta dos persistentes esforços do indivíduo em se adaptar a sua situação existencial ou a alguma experiência que gera sentimentos de tensão, ansiedade, medo ou ameaça, que pode ser de origem interna ou externa. O estresse é quase sempre visualizado como algo negativo que ocasiona prejuízo no desempenho global do indivíduo. No entanto, o estresse nem sempre é um fator de desgaste emocional e físico, mas, sim, um mecanismo natural de defesa do organismo ${ }^{11,12}$.

A depressão, a ansiedade e o estresse estão associados à diminuição da produtividade, do desempenho no trabalho, da qualidade de vida e da saúde dos trabalhadores, além de limitar a contribuição que o portador de seus sintomas poderia dar à sociedade, dessa maneira, causando impacto na vida do indivíduo que a desenvolve ${ }^{13}$. A atividade docente associada a condições precárias de trabalho pode ser fator importante no desencadeamento de doenças, além de provocar o afastamento do docente devido à perda de capacidade física e psíquica para desenvolver suas atividades ${ }^{14}$.

Diante dos problemas e demandas atuais, esses profissionais precisam de competência pedagógica, social e emocional para estimular a construção crítica dos indivíduos para que aprendam a ser e a conviver na sociedade como sujeitos conscientes, reflexivos e participativos, mas, para isso, é fundamental que também estejam física e mentalmente saudáveis ${ }^{5}$.

No que se refere à depressão, à ansiedade e ao estresse, essas patologias têm se agravado no período da pandemia da COVID-19. Os professores tiveram que reinventar a forma de ensinar, considerando o exercício do trabalho remoto, que evidenciou a precarização da atividade docente, com aumento da carga horária on-line involuntária, já que há necessidade de o docente estar conectado e envolvido com suas atividades por um período de tempo muito maior e sem remuneração prevista ${ }^{15}$. Estudos demonstram que essa condição impacta não apenas as dimensões financeiras, afetivas e éticas dos docentes, mas também as dimensões motivacionais, levando ao desânimo, à depressão, à ansiedade e à exaustão ${ }^{15,16}$.

Em 11 de março de 2020, a Organização Mundial da Saúde (OMS) declarou a COVID-19 como uma pandemia ${ }^{17}$. A COVID-19 é uma doença causada pelo coronavírus SARSCoV-2, que apresenta um quadro clínico que varia de infecções assintomáticas a quadros respiratórios graves ${ }^{18,19}$. Sendo assim, o Ministério da Educação, em caráter excepcional, por meio das Portarias nos 343 e 345, de 17 e 19 de março de 2021, respectivamente, autorizou que instituições de educação superior públicas e privadas substituíssem disciplinas presenciais por aulas que utilizem meios e tecnologias de informação e comunicação em cursos que estão em andamento ${ }^{20}$. E de acordo com um noticiário publicado pela Universidade de São Paulo ${ }^{21}$, a partir desse acontecimento, 
tanto os alunos quanto os professores passaram a vivenciar a experiência inédita do ensino remoto em massa e, nesse contexto, o processo de ensino e aprendizagem se tornou complexo, visto que o processo de aprender superou o simples fato de transmitir informações ${ }^{21}$.

É um desafio reconstruir no mundo on-line todas as relações e a estrutura de apoio de uma escola ${ }^{21}$. E isso tem gerado ainda mais estresse e ansiedade entre os docentes do Brasil e do mundo, uma vez que o processo de ensino aprendizagem vai além de disponibilizar os conteúdos de forma on-line, sendo preciso assegurar de forma profícua que as habilidades e competências dos estudantes sejam garantidas; para isso, cabe ao professor se reinventar e planejar as novas metodologias para oferta do conteúdo de forma remota, o que, associado ao isolamento social, acaba por ocasionar ainda mais sintomas de depressão, ansiedade e estresse ${ }^{15,16}$.

Além disso, recentemente, em uma revisão de literatura, apontou-se que são escassos os estudos sobre a relação do sofrimento psíquico e a instabilidade emocional dos docentes em decorrência do ensino remoto emergencial ${ }^{15}$. Sendo assim, estudos que avaliam a prevalência de sofrimento psíquico e os fatores associados nessa população se tornam relevantes. Nesse contexto, o presente estudo buscou estimar a prevalência e os fatores associados aos sintomas da depressão, ansiedade e estresse em professores universitários da área da saúde no período da pandemia da COVID-19.

\section{MÉTODOS}

Trata-se de um estudo epidemiológico analítico, de caráter transversal e abordagem quantitativa ${ }^{22}$. A população do estudo foi composta por professores universitários de ambos os sexos, com faixa etária entre 25 e 62 anos de idade, que lecionam em sete cursos superiores da área da saúde de uma instituição de ensino superior (IES) privada da cidade de Montes Claros, norte de Minas Gerais, sendo eles: Biomedicina, Ciências Biológicas (bacharelado), Enfermagem, Farmácia, Nutrição, Odontologia e Psicologia.

Para o cálculo do tamanho amostral, considerou-se o total de professores vinculados à IES no primeiro semestre do ano de 2020 (250), prevalência desconhecida do desfecho $(50 \%)^{23}$, erro tolerável de $5 \%$ e intervalo de confiança de $95 \%$, totalizando uma amostra mínima de 150 docentes universitários. A amostra não sofreu randomização, tendo sido completada à medida que os questionários eram respondidos.

Foram incluídos os docentes dos cursos da área da saúde da IES que estavam em distanciamento social desde o início da pandemia (março/2020), quando as aulas presenciais foram substituídas pelo ensino remoto emergencial. Foi escoIhido esse público-alvo para participar do estudo, uma vez que a literatura evidencia que profissionais da educação e da saúde estão mais expostos aos riscos psicossomáticos ${ }^{4}$. 0 critério de exclusão foi docente em afastamento temporário, em desvio de função ou que no presente momento estavam prestando serviços para a IES que não fosse a docência.

Após a apresentação do projeto de pesquisa e da assinatura do Termo de Concordância Institucional dos gestores da IES autorizando a realização do estudo nas dependências da instituição, o projeto foi submetido ao Comitê de Ética em Pesquisa (CEP) das Faculdades Integradas do Norte de Minas, tendo sido avaliado e aprovado sob o Parecer no 4.195.679 (CAAE 34536820.0.0000.5141).

Após a aprovação do CEP, os docentes foram convidados para participar do estudo. Caso o docente aceitasse, era necessário que ele assinasse o Termo de Consentimento Livre e Esclarecido (TCLE) on-line, no qual estavam descritos o objetivo do estudo, o procedimento de avaliação e o caráter de voluntariedade da participação. Os participantes do estudo que concordaram em participar da presente pesquisa de forma voluntária responderam ao questionário no Formulário Google Forms:

Para coleta de dados on-line, utilizou-se a rede social, de forma pública, WhatsApp ${ }^{\circledR}$ como meio de disseminação ao público-alvo, por meio da qual foi enviado o formulário para 220 docentes. $\mathrm{O}$ instrumento ficou disponível durante sete dias consecutivos, entre os dias 11 e 17 de outubro de 2020; para garantir que apenas os docentes da IES supracitada respondessem ao questionário, ele foi vinculado ao e-mail institucional dos professores, que tinham direito a responder ao instrumento apenas uma vez. Os primeiros 150 docentes que responderam ao questionário foram incluídos na amostra.

Os dados foram coletados utilizando um questionário que abordava aspectos sociodemográficos, econômicos e trabalhistas. Antes do período da coleta de dados, conduziu-se um estudo-piloto com um grupo de 10 professores, que não fizeram parte da amostra final. O estudo-piloto permitiu que fosse o questionário testado na prática. Após essa fase, a pesquisa de campo foi iniciada. Pequenas alterações de conteúdo foram realizadas no questionário.

As variáveis coletadas para caracterizar os aspectos sociodemográficos, econômicos e trabalhistas foram: sexo (feminino; masculino), idade (em anos e posteriormente dicotomizada em <41 anos; $\geq 41$ anos, levando em consideração a média da idade), estado conjugal (com companheiro fixo; sem companheiro fixo), cor da pele (branca; não branca), renda mensal ( $\geq 5$ salários mínimos; $<5$ salários mínimos), titulação máxima (especialização; mestrado/doutorado), área de formação (biológicas/saúde; outras áreas - exatas + humanas + sociais aplicadas), trabalho em mais de uma IES (não; sim) e carga horária de trabalho semanal (<37 horas; $\geq 37$ horas, levando em consideração a média da carga horária). 
A saúde mental foi avaliada pela Escala de Depressão, Ansiedade e Estresse-21 (DASS-21), adaptada e validada para a língua portuguesa por Vignola e Tuci24. A DASS-21 é um instrumento de autorrelato com 21 questões, e sua pontuação é baseada em uma escala do tipo Likert de quatro pontos, variando de 0 (não se aplicou a mim) a 3 (aplicou-se muito), referente ao sentimento da última semana. As perguntas 3, 5, 10, 13, 16, 17 e 21 formam a subescala de depressão. As perguntas 2, 4, 7, 9, 15, 19 e 20 formam a subescala de ansiedade. As perguntas 1, 6, 8, 11, 12, 14 e 18 formam a subescala de estresse. Para a pontuação final, os valores de cada subescala foram somados e multiplicados por dois para corresponder à pontuação da escala original (DASS-42) ${ }^{25}$.

A classificação dos sintomas de estresse foi: 0-10 = normal; 11-18 = leve; 19-26 moderado; 27-34 = severo; 35-42 = extremamente severo. A classificação dos sintomas de ansiedade foi: 0-6 normal; 7-9 = leve; 10-14 = moderado; 1519 = severo; 20-42 = extremamente severo. A classificação dos sintomas de depressão foi: 0-9 = normal; $10-12=$ leve; 13-20 = moderada; $21-17=$ severo; $28-42=$ extremamente severo ${ }^{18}$. Os sintomas da depressão, ansiedade e estresse foram dicotomizados em ausência e presença, e esta última englobou a pontuação que classificou os sintomas em leve, moderado, severo e extremamente severo e foram designados como variáveis dependentes ${ }^{26}$.

Os dados foram analisados com o auxílio dos softwares Microsoft Excel ${ }^{\circ}$ e Statistical Package for Social Sciences (SPSS)', versão 22. Foi realizada análise descritiva para caracterizar as variáveis estudadas. Para apresentação da distribuição e medida de tendência central das variáveis contínuas numéricas, utilizou-se o teste de Kolmogorov-Smirnov para testar a normalidade dos dados. Análises bivariadas foram realizadas para identificar fatores associados à variável resposta por meio do teste qui-quadrado de Person. A magnitude das associações foi estimada a partir das razões de prevalência (RPs). Por se tratar de uma alternativa para a análise de estudos transversais com resultados binários e prevalência do desfecho acima de 10\% ${ }^{27}$, foi utilizada a regressão de Poisson, com variância robusta, para calcular as RPs ajustadas, considerando, de forma conjunta, as variáveis independentes que estiveram associadas com os sintomas da depressão, ansiedade e estresse na análise bivariada, considerando um nível de significância de 20\% ( $p<0,20)$. A seleção das variáveis no modelo foi realizada com o critério backward stepwise. Para a análise final, considerou-se um nível de significância de $p<$ 0,05 ou $5 \%$.

\section{RESULTADOS}

Um total de 150 indivíduos responderam o questionário entre os dias 11 a 17 de outubro de 2020. A média de idade dos participantes foi de 41,4 \pm 7,9 anos, variando entre 25 e 62 anos; a maioria da amostra investigada é do sexo feminino (74\%). A renda média dos professores foi de 5.385,81 $\pm 2.490,5$ reais, o tempo médio docência foi de 11,21 $\pm 4,9$ anos e a carga horária média de trabalho semanal foi de $37,13 \pm 16,6$ horas. Na tabela 1, são encontradas as características sociodemográficas, econômicas e trabalhistas dos participantes do estudo.

A tabela 2 mostra os dados referentes à escala DASS-21. A pontuação mediana dos sintomas de depressão durante o surto da COVID-19 foi 9,0, variando entre 0 e 42 pontos. A mediana de ansiedade foi 6,0, variando entre 0 e 42 . E a mediana de estresse foi 16,0, variando entre 0 e 40. Observou-se que $50 \%$ dos professores apresentaram sintomas de depressão, $34,6 \%$ relataram sintomas de ansiedade e $42,6 \%$ apresentaram sintomas de estresse.

A tabela 3 apresenta os resultados das análises bivariadas dos fatores associados aos sintomas da depressão, ansiedade

Tabela 1. Características sociodemográficas, econômicas e trabalhistas de professores universitários da área da saúde - Montes Claros, MG ( $n=150)$

\begin{tabular}{|c|c|c|}
\hline Variáveis & $\mathbf{n}$ & $\%$ \\
\hline \multicolumn{3}{|l|}{ Sexo } \\
\hline Feminino & 111 & 74,0 \\
\hline Masculino & 39 & 26,0 \\
\hline \multicolumn{3}{|l|}{ Faixa etária } \\
\hline$<41$ anos & 58 & 38,7 \\
\hline$\geq 41$ anos & 92 & 61,3 \\
\hline \multicolumn{3}{|l|}{ Estado civil } \\
\hline Com companheiro fixo & 100 & 66,7 \\
\hline Sem companheiro fixo & 50 & 33,3 \\
\hline \multicolumn{3}{|l|}{ Cor da pele } \\
\hline Branca & 72 & 48,0 \\
\hline Não branca & 78 & 52,0 \\
\hline \multicolumn{3}{|l|}{ Renda } \\
\hline$\geq 5$ salários & 88 & 58,7 \\
\hline$<5$ salários & 62 & 41,3 \\
\hline \multicolumn{3}{|l|}{ Titulação máxima } \\
\hline Especialização & 61 & 40,7 \\
\hline Mestrado/doutorado & 89 & 59,3 \\
\hline \multicolumn{3}{|l|}{ Área de formação } \\
\hline Biológicas/Saúde & 119 & 79,3 \\
\hline Outras áreas & 31 & 20,7 \\
\hline \multicolumn{3}{|c|}{ Trabalha em mais de uma IES } \\
\hline Não & 81 & 54,0 \\
\hline $\operatorname{Sim}$ & 69 & 46,0 \\
\hline \multicolumn{3}{|l|}{ Carga horária semanal } \\
\hline$<37$ horas & 56 & 37,3 \\
\hline$\geq 37$ horas & 94 & 62,7 \\
\hline
\end{tabular}

IES: instituição de ensino superior. 
e estresse avaliados pela escala DASS-21. Verificou-se que as seguintes variáveis se mostraram associadas à depressão ao nível de significância de $p<0,20$ : faixa etária, estado civil, cor da pele, titulação máxima e trabalhar em mais de uma IES. As variáveis que se mostraram associadas à ansiedade foram: faixa etária, estado civil, renda e titulação máxima. Já as

Tabela 2. Descrição das variáveis de depressão, ansiedade e estresse pela escala DASS-21 em professores universitários da área da saúde - Montes Claros, $M G(n=150)$

\begin{tabular}{|c|c|c|c|}
\hline & $\begin{array}{c}\text { DASS-21 } \\
\text { Depressão }\end{array}$ & $\begin{array}{l}\text { DASS-21 } \\
\text { Ansiedade }\end{array}$ & $\begin{array}{l}\text { DASS-21 } \\
\text { Estresse }\end{array}$ \\
\hline Mediana $(Q 1-Q 3)^{*}$ & $9,0(2,0-22,0)$ & $6,0(2,0-22,0)$ & $16,0(9,5-26,0)$ \\
\hline Normal - n(\%) & $75(50,0)$ & $98(65,4)$ & $86(57,4)$ \\
\hline Leve - n(\%) & $09(6,0)$ & $05(3,3)$ & $53(35,3)$ \\
\hline Moderado - n(\%) & $26(17,3)$ & $30(20,0)$ & $11(7,3)$ \\
\hline Severo - n(\%) & $09(6,0)$ & $15(10,0)$ & - \\
\hline Extremamente severo - n(\%) & $31(20,7)$ & $02(1,3)$ & - \\
\hline
\end{tabular}

* $Q 1: 25^{\circ}$ percentil; Q3: $75^{\circ}$ percentil.

Tabela 3. Distribuição (\%) dos sintomas de depressão, ansiedade e estresse segundo as características sociodemográficas, econômicas e trabalhistas Montes Claros, MG $(n=150)$

\begin{tabular}{|c|c|c|c|c|c|c|c|c|c|c|c|c|c|c|c|}
\hline & \multicolumn{4}{|c|}{ Depressão } & \multirow{3}{*}{$\mathbf{p}^{*}$} & \multicolumn{4}{|c|}{ Ansiedade } & \multirow{3}{*}{$\mathbf{p}^{*}$} & \multicolumn{4}{|c|}{ Estresse } & \multirow{3}{*}{$\mathbf{p}^{\star}$} \\
\hline & \multicolumn{2}{|c|}{ Ausência } & \multicolumn{2}{|c|}{ Presença } & & \multicolumn{2}{|c|}{ Ausência } & \multicolumn{2}{|c|}{ Presença } & & \multicolumn{2}{|c|}{ Ausência } & \multicolumn{2}{|c|}{ Presença } & \\
\hline & $\mathbf{N}$ & $\%$ & n & $\%$ & & $\mathbf{n}$ & $\%$ & n & $\%$ & & N & $\%$ & n & $\%$ & \\
\hline Sexo & & & & & 0,852 & & & & & 0,324 & & & & & 0,892 \\
\hline Feminino & 55 & 73,3 & 56 & 74,7 & & 70 & 71,4 & 41 & 78,8 & & 64 & 74,4 & 47 & 73,4 & \\
\hline Masculino & 20 & 26,7 & 19 & 25,3 & & 28 & 28,6 & 11 & 21,2 & & 22 & 25,6 & 17 & 26,6 & \\
\hline Faixa etária & & & & & $0,094^{\mathrm{a}}$ & & & & & $0,005^{a}$ & & & & & 0,445 \\
\hline$<41$ anos & 24 & 32,0 & 34 & 45,3 & & 30 & 30,6 & 28 & 53,8 & & 31 & 36,0 & 27 & 42,2 & \\
\hline$\geq 41$ anos & 51 & 68,0 & 41 & 54,7 & & 68 & 69,4 & 24 & 46,2 & & 55 & 64,0 & 37 & 57,8 & \\
\hline Estado civil & & & & & $0,166^{a}$ & & & & & $0,185^{a}$ & & & & & $0,062^{\mathrm{a}}$ \\
\hline Com companheiro fixo & 46 & 61,3 & 54 & 72,0 & & 62 & 63,3 & 38 & 73,1 & & 52 & 60,5 & 48 & 75,0 & \\
\hline Sem companheiro fixo & 29 & 38,7 & 21 & 28,0 & & 36 & 36,7 & 14 & 26,9 & & 34 & 39,5 & 16 & 25,0 & \\
\hline Cor da pele & & & & & $0,102^{\mathrm{a}}$ & & & & & 0,501 & & & & & $0,059^{a}$ \\
\hline Branca & 41 & 54,7 & 31 & 41,3 & & 49 & 50,0 & 23 & 44,2 & & 47 & 54,7 & 25 & 39,1 & \\
\hline Não branca & 34 & 45,3 & 44 & 58,7 & & 49 & 50,0 & 29 & 55,8 & & 39 & 45,3 & 39 & 60,9 & \\
\hline Renda & & & & & 0,507 & & & & & $0,122^{a}$ & & & & & 0,879 \\
\hline$\geq 5$ salários & 46 & 61,3 & 42 & 56,0 & & 61 & 62,2 & 27 & 51,9 & & 50 & 58,1 & 38 & 59,4 & \\
\hline$<5$ salários & 29 & 38,7 & 33 & 44,0 & & 37 & 37,8 & 25 & 48,1 & & 36 & 41,9 & 26 & 40,6 & \\
\hline Titulação máxima & & & & & $0,013^{\mathrm{a}}$ & & & & & $0,032^{a}$ & & & & & 0,730 \\
\hline Especialização & 38 & 50,7 & 23 & 30,7 & & 46 & 46,9 & 15 & 28,8 & & 36 & 41,9 & 25 & 39,1 & \\
\hline Mestrado/doutorado & 37 & 49,3 & 52 & 69,3 & & 52 & 53,1 & 37 & 71,2 & & 50 & 58,1 & 39 & 60,9 & \\
\hline Área de formação & & & & & 0,840 & & & & & 0,595 & & & & & 0,753 \\
\hline Biológicas/saúde & 59 & 78,7 & 60 & 80,0 & & 79 & 80,6 & 40 & 76,9 & & 69 & 80,2 & 50 & 78,1 & \\
\hline Outras áreas & 16 & 21,3 & 15 & 20,0 & & 19 & 16,4 & 12 & 23,1 & & 17 & 19,8 & 14 & 21,9 & \\
\hline Trabalha em mais de uma IES & & & & & $0,140^{\mathrm{a}}$ & & & & & 0,474 & & & & & $0,030^{\mathrm{a}}$ \\
\hline Não & 45 & 60,0 & 36 & 48,0 & & 55 & 56,1 & 26 & 50,0 & & 53 & 61,6 & 28 & 43,8 & \\
\hline Sim & 30 & 40,0 & 39 & 52,0 & & 43 & 43,9 & 26 & 50,0 & & 33 & 38,4 & 36 & 56,2 & \\
\hline Carga horária semanal & & & & & 0,736 & & & & & 0,359 & & & & & 0,323 \\
\hline$<37$ horas & 29 & 38,7 & 27 & 36,0 & & 34 & 34,7 & 22 & 42,3 & & 35 & 40,7 & 21 & 32,8 & \\
\hline$\geq 37$ horas & 46 & 61,3 & 48 & 64,0 & & 64 & 65,3 & 30 & 57,7 & & 51 & 59,3 & 43 & 67,2 & \\
\hline
\end{tabular}

* Teste qui-quadrado de Pearson; $p$ valor = nível de significância $p<0,20$; ${ }^{2}$ variáveis selecionadas para o modelo final considerando o nível de significância $p<0,20$. IES: instituição de ensino superior. 
variáveis que se associaram ao estresse foram: estado civil, cor da pele e trabalhar em mais de uma IES.

Após análise múltipla, observou-se que os sintomas da depressão estiveram associados à variável trabalhar em mais de uma IES. As variáveis que se mostraram associadas à ansiedade foram: faixa etária $\geq 40$ anos e pessoas sem companheiro fixo. Já o estresse se mostrou associado à variável estado civil sem companheiro fixo (Tabela 4).

\section{DISCUSSÃO}

Os resultados apontam que uma parcela considerável dos professores analisados apresentou sintomas de depressão, ansiedade e estresse. Estudos conduzidos em diferentes países utilizando o mesmo instrumento ${ }^{28-30}$ apresentaram prevalência de sintomas de depressão, ansiedade e estresse inferiores aos nossos achados. Entretanto, a justificativa para esses achados é que os estudos foram realizados no início do distanciamento social e que a prevalência de sintomas psicossomáticos tende a aumentar com o confinamento ${ }^{29}$.

A literatura aponta que a prevalência desses sintomas psicossomáticos, como depressão, ansiedade e estresse, em professores universitários é alta ${ }^{31-33}$ e que esses fatores têm se agravado durante a pandemia da COVID-19 ${ }^{16}$. Em um estudo com 43.845 professores mexicanos, os resultados apontaram que $16 \%$ da população analisada apresentou sintomatologia para depressão grave ${ }^{31}$. Estudo realizado em uma IES em uma capital do Nordeste do Brasil, observou que 100\% dos professores apresentaram algum nível de depressão ${ }^{32}$, uma

Tabela 4. Fatores associados aos sintomas de depressão, ansiedade e estresse em professores universitários da área da saúde (análise múltipla)

\begin{tabular}{|c|c|c|c|c|c|c|}
\hline & \multicolumn{2}{|c|}{ Depressão } & \multicolumn{2}{|c|}{ Ansiedade } & \multicolumn{2}{|c|}{ Estresse } \\
\hline & $\mathbf{R P}_{\text {bruta }}($ IC95\%) & $\mathbf{R P}_{\text {ajustada }}(\mathbf{I C 9 5 \% )}$ & $\mathbf{R P}_{\text {bruta }}($ IC95\%) & $\mathbf{R P}_{\text {ajustada }}$ (IC95\% & $\mathbf{R P}_{\text {bruta }}$ (IC95\%) & $\mathrm{RP}_{\text {ajustada }}$ (IC95\%) \\
\hline \multicolumn{7}{|l|}{ Sexo } \\
\hline Feminino & 1 & - & 1 & - & 1 & - \\
\hline Masculino & $1,03(0,71-1,50)$ & - & $1,31(0,75-2,28)$ & - & $0,97(0,64-1,47)$ & - \\
\hline \multicolumn{7}{|l|}{ Faixa etária } \\
\hline$<41$ anos & 1 & - & 1 & 1 & 1 & - \\
\hline$\geq 41$ anos & $1,31(0,96-1,80)$ & - & $1,85(1,19-2,85)$ & $1,79(1,07-3,00)$ & $1,15(0,79-1,67)$ & - \\
\hline \multicolumn{7}{|l|}{ Estado civil } \\
\hline Com companheiro fixo & 1 & - & 1 & 1 & 1 & 1 \\
\hline Sem companheiro fixo & $1,28(0,88-1,86)$ & - & $1,35(0,81-2,26)$ & $1,65(1,01-2,69)$ & $1,50(0,95-2,35)$ & $1,76(1,36-3,34)$ \\
\hline \multicolumn{7}{|l|}{ Cor da pele } \\
\hline Branca & 1 & - & 1 & - & 1 & - \\
\hline Não branca & $0,76(0,54-1,06)$ & - & $0,85(0,55-1,33)$ & - & $0,69(0,47-1,02)$ & - \\
\hline \multicolumn{7}{|l|}{ Renda } \\
\hline 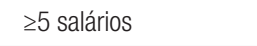 & 1 & - & 1 & - & 1 & - \\
\hline$<5$ salários & $0,89(0,65-1,23)$ & - & $0,76(0,49-1,17)$ & - & $1,03(0,70-1,50)$ & - \\
\hline \multicolumn{7}{|l|}{ Titulação máxima } \\
\hline Especialização & 1 & - & 1 & - & 1 & - \\
\hline Mestrado/Doutorado & $0,64(0,44-0,93)$ & - & $0,59(0,35-0,97)$ & - & $0,93(0,63-1,37)$ & - \\
\hline \multicolumn{7}{|l|}{ Área de formação } \\
\hline Biológicas/Saúde & 1 & - & 1 & - & 1 & - \\
\hline Outras áreas & $1,04(0,69-1,56)$ & - & $0,86(0,52-1,44)$ & - & $0,93(0,59-1,44)$ & - \\
\hline \multicolumn{7}{|c|}{ Trabalha em mais de uma IES } \\
\hline Não & 1 & 1 & 1 & - & 1 & - \\
\hline Sim & $0,78(0,57-1,08)$ & $1,31(1,11-1,57)$ & $0,85(0,54-1,32)$ & - & $0,66(0,45-0,96)$ & - \\
\hline \multicolumn{7}{|l|}{ Carga horária semanal } \\
\hline$<37$ horas & 1 & - & 1 & - & 1 & - \\
\hline$\geq 37$ horas & $0,94(0,67-1,32)$ & - & $1,23(0,79-1,91)$ & - & $0,82(0,54-1,22)$ & - \\
\hline \multicolumn{7}{|l|}{ Estado de saúde } \\
\hline Positiva & 1 & - & 1 & - & 1 & - \\
\hline Negativa & $0,72(0,47-1,12)$ & - & $1,04(0,45-2,39)$ & - & $0,60(0,38-0,95)$ & - \\
\hline
\end{tabular}

RP: razão de prevalência; IC95\%: intervalo de confiança de 95\%; IES: instituição de ensino superior. 
proporção duas vezes maior que os resultados do presente estudo.

Os sintomas da ansiedade também têm sido prevalentes entre os professores universitários. Estudo realizado com professores universitários no Reino Unido encontrou prevalência de 31,6\% ansiedade ${ }^{33}$, resultado semelhante aos achados do presente estudo. No Brasil, estudo que investigou docentes de uma IES da capital mineira observou que $42,7 \%$ da amostra apresentou sinais de ansiedade ${ }^{34}$. E pesquisa com professores universitários egípcios demonstrou que 67,5\% dos investigados apresentaram algum grau de ansiedade ${ }^{35}$, quase o dobro da prevalência encontrada neste estudo.

O estresse também tem sido amplamente relatado na literatura, e estudo realizado com professores da Nigéria com o objetivo de avaliar a prevalência de estresse em professores observou que $72 \%$ dos investigados apresentaram sinais de estresse ${ }^{36}$, resultado superior aos achados do presente estudo. No Brasil, estudo realizado com professores universitários de uma instituição privada do interior de Minas Gerais observou que $36,7 \%$ dos investigados apresentavam sintomas de estresse ${ }^{37}$. Outro estudo realizado com professores de uma IES no Rio de Janeiro observou que 46\% dos investigados possuem sintomas de estresse, segundo o Inventário de Sintomas de Stress para Adultos de Lipp ${ }^{38}$, resultados semelhantes aos achados do presente estudo.

Os sintomas de depressão, ansiedade e estresse têm aumentado entre os docentes no período da pandemia da COVID-1915,16,29. Dentre os motivos citados na literatura pelos quais a prevalência de sintomas psicossomáticos em professores tem aumentado, destaca-se o distanciamento social, como um importante preditor ${ }^{16,29}$. Revisão integrativa da literatura que buscou refletir a respeito das experiências do ensino remoto emergencial pelo corpo docente universitário e dos impactos na saúde mental desses profissionais durante a pandemia da COVID-19 aponta também que, no caso dos docentes universitários, as novas exigências educacionais têm levado a um aumento da carga horária on-line involuntária, estando o docente conectado e envolvido com suas atividades por um período de tempo muito maior e sem remuneração prevista ${ }^{15}$.

Estudos revelam que o excesso de trabalho, muitas vezes associado à qualidade de vida ruim dos profissionais docente, pode ser um dos fatores que têm agravado esse quadro de adoecimento entre professores ${ }^{5,13,39}$, aumentando de forma considerável a prevalência de sintomas de depressão, ansiedade e estresse no Brasi $\left.\right|^{32,34,37,38}$ e no mundo $0^{31,33,35,36}$.

Além disso, o modelo empresarial de ensino, característico das organizações de ensino superior privadas, impacta não apenas as dimensões financeiras, afetivas e éticas dos docentes, como também as dimensões motivacionais, levando ao desestímulo, à depressão, à ansiedade e à exaustão ${ }^{15}$.

As instituições de ensino, sobretudo as privadas, realizam tarefas muito exigentes em um local de trabalho caracterizado por um ambiente psicossocial crítico. São locais de trabalho intensivos em conhecimento, caracterizados por um ambiente psicossocial crítico, onde a qualidade do desenvolvimento e da transmissão do conhecimento depende principalmente da saúde e do bem-estar do corpo docente, o que pode gerar pressão laboral, ocasionando sintomas de ansiedade e estresse, que podem desencadear sintomas depressivos ${ }^{40}$. Essa condição leva ao afastamento do docente, o que causa prejuízos no desenvolvimento das aulas e implica desorganização na sistemática da instituição, impactando o desempenho do aluno ${ }^{39}$.

Entre as variáveis que se associaram aos sintomas da depressão, observa-se que trabalhar em mais de uma instituição se manteve associada a maior prevalência de sintomas de depressão. Um dos fatores que influenciam os professores do ensino superior a trabalharem em mais de uma IES é o fato de que muitas vezes a remuneração que eles recebem não é suficiente para eles arcarem com as despesas, então eles optam por exercer atividades profissionais em mais de um local. Isso faz com que os professores se sintam obrigados a atender às demandas das instituições dentro e fora da sala de aula, o que faz com que, consequentemente, aumente a cobrança e diminua o tempo livre e disponível para lazer ${ }^{41}$.

Apesar de a variável carga horária de trabalho não ter permanecido no modelo final, sabe-se que trabalhar em mais de uma IES é um fator que aumenta a carga horária de trabalho, uma vez que o professor precisa preparar aulas, avaliações, correções das avaliações e reuniões ${ }^{42}$, o que muitas vezes é uma carga horária não contabilizada. No período da pandemia, essa situação se agravou, pois os professores tiveram que modificar suas práticas pedagógicas, e houve a necessidade de gravação das videoaulas, além das aulas remotas, que acontecem ao vivo, com pouca participação dos alunos, e muitas vezes o professor se sente pressionado e com a necessidade de fazer algo para chamar a atenção dos alunos.

Quanto à ansiedade, observou-se que pessoas com idade $\geq 40$ anos estão sujeitas a maior prevalência de sintomas de ansiedade. Estudo realizado com professores no interior de São Paulo observou associação de níveis de ansiedade com os sujeitos que apresentavam idade superior a 40 anos, e mais de $70 \%$ dos questionados apresentaram algum nível de ansiedade ${ }^{14}$. Souza et al. ${ }^{43}$ também afirmam que a prevalência de sintomas depressivos e/ou ansiosos tendem a aumentar com o avanço da idade, atingindo picos elevados após os 40 anos.

No que se refere a sintomas de ansiedade em professores, a literatura aponta que níveis elevados dessa patologia podem comprometer o processo de ensino-aprendizagem. E, após os 40 anos, as pessoas deixam de se perceber como jovens, o que pode influenciar nos seus sentimentos, e deixam de realizar certas atividades e ações, o que leva ao aumento da ansiedade, e isso acaba refletindo na sua atuação profissional ${ }^{14}$. 
Outro fator que se associou à ansiedade e ao estresse foi o estado civil. Entre os professores que relataram não possuir companheiro fixo, foi observada maior prevalência de sintomas de ansiedade e estresse; possivelmente, a desvalorização cultural e a falta de apoio cotidiano mais próximo justificam esse resultado ${ }^{43}$. Ter companheiro diminui o risco de desenvolver desordens mentais, como ansiedade e estresse, uma vez que os profissionais casados tendem a ser mais maduros e estáveis; ademais, com o convívio familiar, o indivíduo desenvolve relações interpessoais, além de priorizar a segurança em detrimento da satisfação pessoal e da famíliaa ${ }^{44,45}$.

No presente estudo, a variável cor da pele mostrou-se associada aos sintomas da depressão e estresse apenas na análise bivariada. Entretanto, em uma revisão sistemática, observou-se que a prevalência de transtornos mentais foi maior na população não branca, quando comparada com a população branca, sugerindo que há relação entre a raça/cor não branca e transtornos mentais, mesmo após controle de variáveis socioeconômicas, como escolaridade ou renda familiar ${ }^{46}$.

A população não branca brasileira ocupou historicamente as classes sociais mais pobres e de condições mais precárias na pirâmide social. As condições indignas de vida da população não branca no Brasil persistem, evidenciando o racismo silencioso e não declarado, fator que pode concorrer para o adoecimento psíquico dessa população. A relação entre raça e saúde, de maneira geral, e, mais ainda, entre raça e saúde mental especificamente, é tema negligenciado na literatura brasileira, entretanto parece clara a existência do impacto do racismo na saúde mental e no adoecimento das pessoas que sofrem preconceito, como no caso das pessoas não brancas ${ }^{47}$.

Embora a titulação do professor não tenha se mostrado significante na análise final, essa característica tem sido discutida na literatura como um fator que pode levar a maior frequência de adoecimento. Destaca-se que cursar uma pós-graduação stricto sensu é um fator que aumenta o risco de depressão, ansiedade e estresse, tendo sido apontado que o sofrimento faz parte do processo de formação de professores e pesquisadores, e manifesta-se em diversos níveis de intensidade ${ }^{48-50}$.

Ao cursar um mestrado ou doutorado, é comum a exposição a fatores que podem desencadear transtornos psíquicos e que se fazem presentes durante a sua formação, entre os quais podem ser citados a sobrecarga de atividades curriculares, os níveis altos de atuação e exigências, a falta de tempo e a dificuldade de otimização desse tempo entre os estudos e a vida particular ${ }^{50}$. Nesse contexto, levando em consideração a exigência de que os professores universitários tenham a titulação de mestre ou doutor e associando a vida profissional com a formação acadêmica, sugere-se que a associação entre os sintomas da depressão e ansiedade com a titulação dos professores seja justificada pelas condições de trabalho dos professores, mais as exigências com o processo de formação, desencadeando, assim, os transtornos psíquicos.

Pela natureza de inquéritos epidemiológicos, este estudo possui algumas limitações, podendo-se destacar alguns itens das perguntas que constituíram o instrumento de coleta de dados, que, mesmo que tenham sido validadas, podem ter sido mal compreendidas ou respondidas distorcidamente, uma vez que a coleta foi baseada em questionários autoaplicáveis, não sendo também suficiente para confirmar diagnósticos de problemas mentais. Outra limitação diz respeito ao fato de não ter sido feita a randomização da amostra, o que possibilita viés de seleção, sobretudo se considerarmos que pessoas com problemas de saúde mental poderiam ser menos propensas a responder o questionário, levando à subestimação do problema aqui tratado, que já apresenta valores elevados. Contudo, mesmo com limitações, destaca-se que esta investigação trata de um assunto importante e negligenciado, especialmente no contexto da pandemia da COVID-19: a saúde mental de professores universitários da área da saúde. Dessa forma, este trabalho pode auxiliar no direcionamento da atenção diante dessa situação.

\section{CONCLUSÃO}

A prevalência de sintomas de depressão, ansiedade e estresse em professores universitários da área da saúde foi elevada, e fatores sociodemográficos (faixa etária e estado civil) e trabalhistas (trabalhar em mais de uma instituição) mantiveram-se associados aos desfechos investigados, o que indica a necessidade de valorizar o estado de saúde mental dos professores. Dessa forma, o desenvolvimento de estratégias pode auxiliar direta ou indiretamente na redução da prevalência da sintomatologia de depressão, ansiedade e estresse e, consequentemente, melhorar a qualidade de vida e o desempenho desses professores universitários.

\section{CONTRIBUIÇÕES INDIVIDUAIS}

Ronilson Ferreira Freitas - Análises estatísticas, interpretação dos dados, auxílio na construção dos itens, revisão crítica do conteúdo intelectual e aprovação da versão final para submissão.

Daniel Santos Ramos - Construção dos itens, referencial teórico, coleta de dados, escrita do artigo e aprovação da versão final para submissão.

Tahiana Ferreira Freitas - Construção dos itens, referencial teórico, coleta de dados, escrita do artigo e aprovação da versão final para submissão.

Gleydson Rocha de Souza - Construção dos itens, referencial teórico, coleta de dados, escrita do artigo e aprovação da versão final para submissão. 
Éryka Jovânia Pereira - Construção dos itens, referencial teórico, coleta de dados, escrita do artigo e aprovação da versão final para submissão.

Angelina do Carmo Lessa - Análises estatísticas, interpretação dos dados, auxílio na construção dos itens, revisão crítica do conteúdo intelectual e aprovação da versão final para submissão.

\section{CONFLITO DE INTERESSES}

Nenhum dos autores apresentou divergência de interesses ao longo da elaboração do projeto e execução do trabalho.

\section{REFERÊNCIAS}

1. Costa DS, Medeiros NSB, Cordeiro RA, Frutuoso ES, Lopes JM, Moreira SNT. Sintomas de Depressão, Ansiedade e Estresse em Estudantes de Medicina e Estratégias Institucionais de Enfrentamento. Rev Bras Educ Méd. 2020:44(11):e040.

2. Parreira BDM, Goulart BF, Ruiz MT, Silva SR, Gomes-Sponhols FA. Depression symptoms in rural women: sociodemographic, economic, behavioral, and reproductive factors. Acta Paul Enferm. 2017;30(4):375-82.

3. Assumpção GLS, Oliveira LA, Souza MFS. Depressão e suicídio: uma correlação. Pretextos - Revista da Graduação em Psicologia da PUC Minas. 2018;3(5).

4. Ferreira RC, Silveira AP, Sá MAB, Feres SBL, Souza JG, Martins AMEBL. Transtorno mental e estressores no trabalho entre professores universitários da área da saúde. Trab Educ Saúde. 2015;13(Supl 1):135-55.

5. Diehl L, Marin AH. Adoecimento mental em professores brasileiros: revisão sistemática da literatura. Est Inter Psicol. 2016;7(2):64-85.

6. Monteiro LCC, Lage AMV. Depressão - uma psicopatologia classificada nos Manuais de Psiquiatria. Psicol Cienc Prof. 2002;27(1):106-19.

7. American Psychiatric Association (APA). Manual Diagnóstico e Estatístico de Transtornos Mentais: DSM-IV. 4a ed. Porto Alegre: Artes Médicas; 1995

8. Organização Mundial da Saúde (OMS). Classificação dos Transtornos Mentais e de Comportamento da CID-10. Porto Alegre: Artes Médicas; 1993.

9. Barros ALBL, Humerez DC, Fakih FT, Michel JLM. Situações geradoras de ansiedade e estratégias para seu controle entre enfermeiras: estudo preliminar. Rev Latino-Am Enfermagem. 2003;11(5):585-92.

10. Carvalho R, Farah OGD, Galdeano LE. Níveis de ansiedade de alunos de graduação em enfermagem frente à primeira instrumentação cirúrgica. Rev Latino-Am Enfermagem. 2004;12(6):918-23

11. Stacciarini JM, Tróccoli BT. 0 estresse na atividade ocupacional do enfermeiro. Rev LatinoAm Enfermagem. 2001;9(2):17-25

12. Favassa (TA, Armiliato N, Kalinine I. Aspectos fisiológicos e psicológicos do Estresse. Rev Psicol UnC. 2005;2(2):84-92.

13. Fernandes MA, Ribeiro HKP, Santos JDM, Monteiro CFS, Costa RS, Soares RFS. Prevalence of anxiety disorders as a cause of workers' absence. Rev Bras Enferm. 2018;71(5):2344-51.

14. Ferreira-Costa RQ, Pedro-Silva N. Levels of anxiety and depression among early childhood education and primary education teachers. Pro-Posições. 2019;30:e20160143.

15. Santos GMRS, Silva ME, Belmonte BR. COVID-19: emergency remote teaching and university professors' mental health. Rev Bras Saúde Matern Infant. 2021;21(Supl 1):S23743.

16. Cruz RM, Rocha RER, Adreoni S, Pesca AD. Retorno ao trabalho? Indicadores de saúde mental em professores durante a pandemia da COVID-19. Polyphonía. 2020;31(1):325-44.

17. World Health Organization. Coronavirus disease 2019: situation Report-46. Geneva; 2020. Disponivel em: https://www.who.int/docs/default-source/coronaviruse/situation- reports/20200306-sitrep-46-covid-19.\%20pdf?sfvrsn=96b04adf_2. Acesso em: 12 jun. 2020.

18. Lescure FX, Bouadma L, Nguyen D, Parisey M, Wicky PH, Behillil S, et al. Clinical and virological data of the first cases of COVID-19 in Europe: a case series. Lancet Infect Dis. 2020;20(6):697-706.

19. Wu F, Zhao S, Yu B, Chen YM, Wang W, Zhi-Gang C, et al. A new coronavirus associated with human respiratory disease in China. Nature. 2020;579(7798):265-9.

20. Brasil. Ministério da Educação. Conselho Nacional de Educação esclarece principais dúvidas sobre 0 ensino no país durante pandemia do coronavírus. Disponível em: http://portal. mec.gov.br/busca-geral/12-noticias/acoes-programas-e-projetos-637152388/87161conselho-nacional-de-educacao-esclarece-principais-duvidas-sobre-0-ensino-no-paisdurante-pandemia-do-coronavirus. Acesso em: 8 jun. 2020.

21. Universidade de São Paulo (USP). Um guia para sobreviver à pandemia do ensino remoto. Disponível em: http://www.saocarlos.usp.br/um-guia-para-sobreviver-a-pandemia-doensino-remoto/. Acesso em: 8 jun. 2020.

22. Lima-Costa MF, Barreto SM. Tipos de estudos epidemiológicos: conceitos básicos e aplicações na área do envelhecimento. Epidemiol Serv Saúde. 2003;12:189-201.

23. Szwarcwald CL, Damacena GN. Amostras complexas em inquéritos populacionais: planejamento e implicações na análise estatística dos dados. Rev Bras Epidemiol. 2008;11(1):38-45.

24. Vignola RCB, Tucci AM. Adaptation and validation of the depression, anxiety and stress scale (DASS) to Brazilian Portuguese. J Affect Disord. 2014;155:104-9.

25. Saadi TA, Addeen SZ, Turk T, Abbas F, Alkhatib M. Psychological distress among medical students in conflicts: A cross-sectional study from Syria. BMC Med Educ. 2017;17(1):173.

26. Corrêa CA, Verlengia R, Ribeiro AGSV, Cris AH. Níveis de estresse, ansiedade, depressão e fatores associados durante a pandemia de COVID-19 em praticantes de Yoga. Rev Bras Ativ Fis Saúde. 2020;25:e0118.

27. Barros AJD, Hirakata VN. Alternatives for logistic regression in cross-sectional studies: an empirical comparison of models that directly estimate the prevalence ratio. BMC Med Res Methodol. 2003;3:21.

28. Kazmi SSH, Hasan K, Talib S, Saxena S. COVID-19 and Lockdown: A Study on the Impact on Mental Health. SSRN Electron J. 2020.

29. Ozamiz-Etxebarria N, Dosil-Santamaria M, Picaza-Gorrochategui M, Idoiaga-Mondragon N. Stress, Anxiety and Depression Levels in the Initial Stage of the COVID-19 Outbreak in a Population Sample in the Northern Spain. Cad Saude Publica. 2020;36(4):e00054020.

30. Wang C, Pan R, Wan X, Tan Y, Xu L, Ho CS, et al. Immediate psychological responses and associated factors during the initial stage of the 2019 coronavirus disease (COVID-19) epidemic among the general population in China. Int J Environ Res Public Health. 2020;17(5):1729.

31. Soria-Saucedo R, Lopez-Ridaura R, Lajous M, Wirtz V. The prevalence and correlates of severe depression in cohort of Mexican teachers. J Affect Disord. 2018;234:109-16.

32. Rodrigues LTM, Lago EC, Almeida CAPL, Ribeiro IP, Mesquita GV. Stress and depression in teachers from a public education institution. Enfermería Global. 2020;19(57):232-42.

33. Mark G, Smith A. Effects of occupational stress, job characteristics, coping, and attributional style on the mental health and job satisfaction of university employees. Anxiety Stress Coping. 2012;25(1):63-78.

34. Alvim AL, Ferrarezi JAS, Silva LM, Floriano LF, Rocha RLP. 0 estresse em docentes de ensino superior. Braz J Develop. 2019;5(12):32547-58.

35. Desouky D, Allam H. Occupational stress, anxiety and depression among Egyptian teachers. J Epidemiol Glob Health. 2017;7:191-8.

36. Asa FT, Lasebikan V0. Mental Health of Teachers: Teachers' Stress, Anxiety and Depression among Secondary Schools in Nigeria. Int Neuropsychiatr Dis J. 2016;7(4):1-10.

37. Cruvinel Júnior RH, Pfister APL, Ferreira EK, Guimarães JC. Níveis de estresse e variabilidade da frequência cardíaca em professores universitários. Revista Interdisciplinar de Estudos em Saúde da UNIARP. 2019;9(2):73-82.

38. Santos MPG, Silva KKD. Níveis de estresse e qualidade de vida de professores do ensino superior. Rev Enferm UFSM. 2017;7(4):656-68.

39. Silva TR, Carvalho EA. Depressão em professores universitários: uma revisão da literatura brasileira. Rev Uningá Rev. 2016;28(1):113-7. 
40. Converso D, Sottimano I, Molinengo G, Loera B. The Unbearable Lightness of the Academic Work: The Positive and Negative Sides of Heavy Work Investment in a Sample of Italian University Professors and Researchers. Sustainability. 2019;11(8):24-39.

41. Monteiro JK, Brun LG, Santos AS, Tundis AG0. Distúrbios Psiquiátricos Menores e Fatores Associados em Professores do Ensino Privado do Rio Grande do Sul/Brasil. Contextos Clínicos. 2019;12(3).

42. Guareschi PA, Guareschi NMF, Genro MEH. Sob a espada de Dâmocles. In: Fuhr MJ (Org.). Sob a espada de Dâmocles: relação dos professores com a docência e ambiente de trabalho no ensino privado. Porto Alegre: Carta Editora; 2013. p. 20-8.

43. Souza GNP, Alves RJR, Souza LPS, Rosa AJ. Prevalência de sintomas depressivos e/ou ansiosos em pessoas com hipertensão arterial sistêmica e/ou diabetes mellitus. Rev Port Enferm Saúde Mental. 2018;20:43-50.

44. Shanafelt TD, Boone S, Tan L, Dyrbye L, Sotile W, Satele D, et al. Burnout and Satisfaction With Work-Life Balance Among US Physicians Relative to the General US Population. Arch Intern Med. 2012;172(18):1377-85.
45. Lima AS, Farah BF, Bustamante-Teixeira MT. Análise da prevalência da síndrome de Burnout em profissionais da Atenção Primária em Saúde. Trab Educ Saúde. 2018;16(1):283-304.

46. Smolen JR, Araújo EM. Race/skin color and mental health disorders in Brazil: a systematic review of the literature. Ciênc Saúde Coletiva. 2017;22(12):4021-30.

47. Damasceno MG, Zanello VML. Saúde mental e racismo contra negros: produção bibliográfica brasileira dos últimos quinze anos. Psico Ciênc Prof. 2018;38(3):450-64.

48. Louzada RCR, Silva Filho JF. Formação do pesquisador e sofrimento mental: um estudo de caso. Psicol Estud. 2005;10(3):451-61.

49. Faro A. Estresse e estressores na pós-graduação: estudo com mestrandos e doutorandos no Brasil. Psic Teor Pesq. 2013;29(1):51-60.

50. Souza JA, Fadel CB, Ferracioli UM. Estresse no cotidiano acadêmico: um estudo com pósgraduandos em Odontologia. Rev ABEN0. 2016;16(1):50-60. 